\title{
Transient Inactivation of the Neonatal Ventral Hippocampus Impairs Attentional Set-Shifting Behavior: Reversal with an $\alpha 7$ Nicotinic Agonist
}

\author{
Julie M Brooks', Michelle L Pershing', Morten S Thomsen², Jens D Mikkelsen², Martin Sarter ${ }^{3}$ and \\ John P Bruno*, I \\ 'Department of Psychology, The Ohio State University, Columbus, OH, USA; ${ }^{2}$ Neurobiology Research Unit, Copenhagen University Rigshospitalet, \\ Copenhagen, Denmark; ${ }^{3}$ Department of Psychology, University of Michigan, Ann Arbor, MI, USA
}

\begin{abstract}
Cognitive deficits represent a core symptom cluster in schizophrenia that are thought to reflect developmental dysregulations within a neural system involving the ventral hippocampus $(\mathrm{VH})$, nucleus accumbens (NAC), and prefrontal cortex (PFC). The present experiments determined the cognitive effects of transiently inactivating $\mathrm{VH}$ in rats during a sensitive period of development. Neonatal (postnatal day 7, PD7) and adolescent (PD32) male rats received a single bilateral infusion of saline or tetrodotoxin (TTX) within the $\mathrm{VH}$ to transiently inactivate local circuitry and efferent outflow. Rats were tested as adults on an attentional set-shifting task. Performance in this task depends upon the integrity of the PFC and NAC. TTX infusions did not affect the initial acquisition or ability to learn an intradimensional shift. However, TTX rats required a greater number of trials than did controls to acquire the first reversal and extradimensional shift (ED) stages. These impairments were age and region-specific as rats infused with TTX into the VH at PD32, or into the dorsal hippocampus at PD7, exhibited performance in the task similar to that of controls. Finally, acute systemic administration of the partial $\alpha 7$ nicotinic acetylcholine receptor (nAChR) agonist SSR 18071 I $(3.0 \mathrm{mg} / \mathrm{kg})$ eliminated the TTX-induced performance deficits. Given that patients with schizophrenia exhibit hippocampal pathophysiology and deficits in the ED stages of set-shifting tasks, our results support the significance of transient hippocampal inactivation as an animal model for studying the cognitive impairments in schizophrenia as well as the pro-cognitive therapeutic potential of $\alpha 7 \mathrm{nAChR}$ agonists.

Neuropsychopharmacology (2012) 37, 2476-2486; doi:I0.1038/npp.20 I2.106; published online II July 2012
\end{abstract}

Keywords: attentional set-shifting; schizophrenia; tetrodotoxin; ventral hippocampus; prefrontal cortex; alpha7 nicotinic acetylcholine receptors.

\section{INTRODUCTION}

Deficits in executive neuronal functions, mediated in large part by the prefrontal cortex (PFC), represent a pervasive core symptom cluster of schizophrenia (SZ; eg, Kerns et al, 2008; Nuechterlein et al, 2009). These cognitive deficits, which are believed to be predictive of the severity of the disorder as well as the functional outcome of the patient (Green et al, 2004), are not effectively alleviated by current pharmacotherapies (Keefe, 2007). Thus, the continued development of animal models of SZ that reproduce corresponding deficits in executive function is vital for the rational discovery of more efficacious treatments.

A main aspect of the cognitive deficits seen in SZ concerns the impairments in the construct of attention,

*Correspondence: Dr JP Bruno, Department of Psychology, The Ohio State University, Columbus, OH 43210, USA. Tel: + I 614 292 1770, Fax: + | 614688 4733. E-mail: bruno. I@osu.edu

Received 19 March 2012; revised 22 May 2012; accepted 26 May 2012 yielding heightened distractibility and cognitive inflexibility (Pantelis et al, 1999; Nuechterlein et al, 2009). Cognitive flexibility, as measured in attentional set-shifting tasks, refers to the ability to shift attentional resources across perceptual attributes of complex, behaviorally relevant environmental stimuli in response to continuous changes in environmental contingencies (Robbins, 2007). As such, cognitive flexibility represents an important property underlying many higher order executive functions (Miller and Cohen, 2001; Robbins, 2007). Clinical evaluations of cognitive flexibility in SZ indicate impairments in their ability to shift attention between perceptual attributes of complex stimuli in response to changing contingencies while concurrently inhibiting previously reinforced responses (Pantelis et al, 1999; Leeson et al, 2009).

Extensive research in rodents indicates that PFCmediated cognitive processes are modulated by a distributed neural system involving multiple forebrain regions including the ventral hippocampus ( $\mathrm{VH})$, nucleus accumbens (NAC), basal forebrain (BF), and the mediodorsal 
nucleus of the thalamus (MD; Brooks et al, 2011; Floresco et al, 2009; Tseng et al, 2009; Hasselmo and Sarter, 2011). Because it is a site of convergence for multiple excitatory inputs from cortical and subcortical regions including the $\mathrm{VH}$ and PFC, the NAC has a central role in the modulation of PFC-related cognitive processing (French and Totterdell, 2003). Through integration of these inputs, the NAC influences PFC activation by various pathways, including direct modulation from the basal forebrain cortical cholinergic system (BFCS; Zaborszky and Cullinan, 1992). We previously demonstrated that glutamatergic excitation of the NAC can evoke acetylcholine ( $\mathrm{ACh}$ ) release in the PFC (Zmarowski et al, 2005; Brooks et al, 2007), thereby recruiting interactions between motivational and attentional mechanisms necessary to ensure stable attentional performance when demands on cognitive control are high (St Peters et al, 2011).

The integrity of the $\mathrm{VH}$ is critical to the functional development and regulation of this distributed neural system. Neurons from the subiculum region of the VH project directly to inhibitory interneurons and pyramidal cells in the PFC (Jay et al, 1989; Tierney et al, 2004) as well as to the NAC (Groenewegen et al, 1987). VH efferent activity is necessary for gating the responsiveness of NAC neurons to PFC input and thus, it regulates the salience of behaviorally relevant information coming from PFC projection neurons (O'Donnell and Grace, 1995). Loss of this VH-mediated gating function leads to impaired information processing within the PFCNAC-BFCS circuit and is hypothesized to yield several of the cognitive deficits seen in SZ (Tseng et al, 2009).

The neonatal ventral hippocampal lesion model (nVHLX) has provided valuable insights into the developmental origins of schizophrenic-like pathophysiology and cognitive deficits (for reviews see Lipska and Weinberger, 2002; Tseng et al, 2009). The nVHLX model, which involves permanent excitotoxic damage to the $\mathrm{VH}$ during a sensitive period in early postnatal development, results in a wide range of neuronal abnormalities hypothesized to contribute to the development of SZ, including: decreases in dendritic spine density in NAC and PFC (Flores et al, 2005); abnormal activation of PFC and NAC neurons in response to mesocorticolimbic stimulation (Goto and O'Donnell, 2002; O'Donnell et al, 2002); and altered regulation of cortical cholinergic activity (Alexander et al, 2009; Brooks et al, 2011; Laplante et al, 2004). The nVHLX model also reveals a range of SZ-like cognitive impairments, including: reductions in prepulse inhibition (Le Pen and Moreau, 2002) and latent inhibition (Grecksch et al, 1999); deficits in working memory (Lipska et al, 2002a; Brady et al, 2010) and cognitive flexibility (Marquis et al, 2008; Brady, 2009) tasks, which are known to be heavily reliant on appropriate hippocampal-PFC-NAC interactions (Chambers et al, 1996; Floresco et al, 2009).

However, the extensive and permanent damage to the hippocampus produced in this animal model far exceeds the modest volume loss and cellular disorganization seen in patients with SZ (Harrison, 2004). As an alternative approach, recent studies have focused on the permanent effects of transient disruption of neuronal transmission in the developing $\mathrm{VH}$ using local administration of tetrodotoxin (TTX), a potent and specific blocker of voltage-gated sodium channels. Several reports suggest that transient inactivation results in developmental perturbations similar to those observed following permanent $\mathrm{VH}$ lesions. These include exaggerated locomotor response to dopamine agonists and glutamate receptor antagonists (Lipska et al, 2002b; Lipska et al, 2003) as well as enduring neurochemical and behavioral dysregulations associated with latent inhibition (Peterschmitt et al, 2008). Most recently, our laboratory has demonstrated deficits in mesolimbic stimulation of prefrontal ACh release identical to those reported in the nVHLX model (Brooks et al, 2011).

The following experiments determined the effects of transient inactivation of the $\mathrm{VH}$, during a critical point in neonatal development (postnatal day 7; PD7), on performance in the attentional set-shifting task first developed by Birrell and Brown (2000). This task is a rodent analogue of intra-dimensional shift (ID)/extra-dimensional shift (ED) cognitive flexibility tasks used in the clinical population, and depends on the functional integrity of the PFC (Robbins, 2007; Floresco et al, 2009; Kehagia et al, 2010; Stefani \& Moghaddam, 2005) and NAC (Robbins, 2007; Floresco et al, 2009). We extended this analysis with two control experiments that addressed the regional specificity of the TTX inactivation (TTX into dorsal hippocampus (DH)) as well as the age dependency of the effect (TTX into $\mathrm{VH}$ at PD32). Finally, we determined whether performance in the attentional set-shifting task could be restored pharmacologically. Based on the well-established role of prefrontal cholinergic activity and attentional processing (for reviews, see Sarter et al, 2005; Hasselmo and Sarter, 2011) and the documented disruption in cortical cholinergic transmission resulting from damage to the developing $\mathrm{VH}$ (Laplante et al, 2004; Alexander et al, 2009), we also determined the pro-cognitive effects of acute systemic administration of SSR180711, an $\alpha 7$ nicotinic acetylcholine receptor ( $\alpha 7 \mathrm{nAChR})$ partial agonist (Biton et al, 2007; Pichat et al, 2007), on the attentional set-shifting deficits seen in this animal model.

\section{MATERIALS AND METHODS}

\section{Subjects}

Adult male Wistar rats (300-400 g body weight) born in our colony (breeders from Charles River Laboratory: Wilmington, MA) and treated with saline or TTX during development, served as subjects in these experiments. Adults were housed individually and were maintained in a temperature $\left(72^{\circ}\right)$ - and humidity (30-70\%)-controlled environment on a $12 \mathrm{~h}$ light (lights on at 0630 hours) : dark cycle with food and water available ad libitum. Animal care and experimentation were performed in accordance with protocols approved by The Ohio State University Institutional Laboratory Animal Care and Use Committee and consistent with the NIH Guide for the Care and Use of Laboratory Animals.

\section{Surgical Procedures}

Transient inactivation of the developing hippocampus.

Neonatal ventral hippocampal inactivation: Neonatal surgical procedures were performed in pups on PD 7 (body weight: $15-18 \mathrm{~g})$. Male pups were selected for these experiments and were randomly assigned to receive saline 
vehicle (nVHSham; $n=7$ ) or TTX (nVHTTX; $n=8$ ) injections. On the day of surgery, pups were anesthetized by hypothermia (placed on ice for 8-10 min) and then secured with tape onto a styrofoam platform that was positioned in a stereotaxic frame. An incision was made in the skin and the ventral hippocampal infusion was made by penetrating the skull with an infusion syringe (10 $\mu$ l syringe, $26 \mathrm{G}$ needle) at coordinates AP $-3.0 \mathrm{~mm}, \mathrm{ML} \pm 3.5 \mathrm{~mm}$, DV $-5.0 \mathrm{~mm}$ relative to Bregma. Saline $(0.9 \%)$ or TTX (Sigma, St Louis, MO; $100 \mu \mathrm{M}$ ) was then infused into the $\mathrm{VH}$ in a total volume of $0.3 \mu \mathrm{l}$ over a 2 -min period. Following completion of the infusion, the needle was left in place for an additional $3 \mathrm{~min}$ to allow diffusion of TTX and prevent backflow up the needle tract. This infusion procedure was then repeated in the contralateral hemisphere. Saline and TTX infusions were tinted with Evans Blue (Sigma; $2.5 \mathrm{mg}$ dye $/ 1 \mathrm{ml}$ saline vehicle) to subsequently localize the site of injection during histological preparations. After surgery, the pups were warmed on a heating pad and returned as a group to the litter's nest area. Pups and dams were left undisturbed until weaning on PD 21. Males treated with TTX or saline were then housed together in groups of 2-3/ cage until being individually housed on PD56. A total of 4 litters were used to generate the 39 subjects for the PD7 VH infusions (including the SSR study). Each litter contributed to the subject pool of each of the four treatment conditions.

In addition, the following two control groups were studied to assess the specificity of the effects seen following the TTX infusions into VH on PD7.

Neonatal dorsal hippocampal inactivation: In these pups (PD7), the DH was infused with TTX (nDHTTX; $n=6$ ) or a saline control (nDHSham; $n=6$ ). Surgical procedures, infusion parameters, and housing conditions were the same as those described for nVHTTX pups, except that the following coordinates were used: $\mathrm{AP}-3.0 \mathrm{~mm}, \mathrm{ML} \pm 2.6$, and DV $-3.2 \mathrm{~mm}$; relative to Bregma. A total of 4 litters were used to generate the 12 subjects in the PD7 DH conditions with each litter contributing pups to both of the two treatment conditions.

Periadolescent ventral hippocampal inactivation: Male rats at PD32 were anesthetized with inhalant isofluorane $\left(2 \%, 0.6 \mathrm{l} / \mathrm{min}, \mathrm{O}_{2}\right)$. TTX $(100 \mu \mathrm{M}, 0.3 \mu \mathrm{l}$;. paVHTTX; $n=7)$ or saline $(0.9 \%$; paVHSham; $n=6)$ was bilaterally infused into the VH (AP: $+3.2 \mathrm{~mm}$ from intra-aural; $\mathrm{L}: \pm 4.4 \mathrm{~mm}$; DV: $-7.2 \mathrm{~mm}$ ) at a rate of $0.15 \mu \mathrm{l} / \mathrm{min}$ with a syringe pump. The needle was left in place for $3 \mathrm{~min}$ and then withdrawn. The incision was closed using silk sutures and the area was swabbed with a topical antibiotic/anaesthetic ointment (Neosporin $+2 \%$ lidocaine). TTX- and saline-treated rats were housed together in groups of 2-3/cage until the time of surgery. Following surgery, the animals were returned to individual home cages and left undisturbed for 28 days. A total of 4 litters were used to generate the 13 subjects for the PD32 VH infusions. Each litter contributed to the subject pool of all four of the treatment conditions.

\section{Attentional Set-Shifting}

Apparatus. Rats were trained to recover a small piece of sugary cereal buried in standard pine chip bedding filling a terra cotta flower pot (height: $3.5 \mathrm{in}$; outer diameter: $4.5 \mathrm{in}$ ). The drainage hole of the pot was filled with clay and the pot was filled to $\frac{3}{4}$ capacity with gardening stones held in place with hardened paraffin. The remaining space from the top of the wax to the top of the pot was left for the addition of distinctive digging media. These pots were used as complex stimuli by varying the material inside the pot (digging media), the material covering the outside of the pot (texture), or the scent added to the digging media (odor).

The set-shifting box in which training and testing were conducted was constructed out of pressed wood $(91 \mathrm{~cm}$ $(\mathrm{L}) \times 40.5 \mathrm{~cm}(\mathrm{~W}) \times 25.4 \mathrm{~cm}(\mathrm{H}))$ covered with self-adhesive, black contact paper. A wood panel divided one-third of the length of the box on one end creating three sections; one large holding area and two equally sized testing arms. The digging pots were placed in these testing sections. A removable divider separated the rat from the two sections holding the pots.

Habituation and training. All training and testing paradigms were modeled after procedures used by Birrell and Brown (2000). Rats were food restricted (given 15-20 g/day) and maintained at a minimum of $85 \%$ of their free-fed body weight for 1 week prior to training/testing. Each day the rats were handled for $5 \mathrm{~min}$ followed by $20 \mathrm{~min}$ of free exploration of the testing environment without the presence of any testing stimuli (ie, pots) and in the absence of the wooden divider. They were also given a piece of cereal in their home cage to familiarize them to the odor and taste of the food reward. During the last day of acclimation, rats were placed in the testing environment with a plain pot filled with unscented wood shavings. Rats were given 20 min to explore the pot and consume food reward placed on top of the wood shavings.

Following the acclimation phase and food restriction, animals began the first of two phases of training occurring on consecutive days. Day 1 training began with rats being placed in the start compartment with the dividing wall in place. The plain pot filled with unscented wood shavings was baited and placed behind the divider in one of the choice arms, out of view of the rat. Upon removal of the divider the rat was given $90 \mathrm{~s}$ to retrieve the reward. The session began with the reward placed on top of the digging medium. Over successive trials, the reward was slowly buried within the medium. Successful completion of this session occurred when the rats were reliably retrieving 10 consecutive, fully buried rewards. To discourage any side bias, the pot was randomly placed in either choice arm over subsequent trials during training Day 1.

During Day 2 training, rats were taught to differentiate among three perceptual dimensions. The rats were presented with pairs of training pots that differed on only one dimension. These included an odor discrimination (lavender $v s$ raspberry), a digging medium discrimination (green shredded paper $v s$ white shredded paper), and a texture discrimination (standard white paper $v s$ parafilm). Throughout the training, the correct pot was baited with the food reward while the incorrect pot contained an equal amount of crushed Fruit Loop at the bottom to prohibit the animal from using the scent of the reward to guide its behavior. Each trial began with the rat confined within 
the larger holding portion of the apparatus with the divider wall in place. The trial began when the divider wall was lifted giving the animal access to the two choice arms, each containing a pot. Rats were initially given four exploration trials $(90 \mathrm{~s}$ each) to explore and dig in both pots until the reward was retrieved. Following the discovery period, trials were continued until the animal reached a response criterion of 10 correct consecutive digs in only the rewarded pot. Training pots were presented in a counter-balanced order across subjects. The exemplars used in training were not used again for testing.

\section{Testing Paradigm}

On Day 3, subjects were tested on a series of discriminations presented in a fixed order for all rats (see Table 1 for stages of the task). Testing began with the presentation of a simple discrimination (SD) with the pots varying along only one dimension. As in training, the first four trials were treated as exploration trials during which the animal was given the opportunity to dig in either the correct or incorrect pot until the reward was retrieved. For the remainder of the testing stage, the rat was given $60 \mathrm{~s}$ to dig in either pot. In the event of an incorrect choice, the divider wall was immediately replaced so that the animal was not allowed access to the alternate pot. If an animal did not dig within $60 \mathrm{~s}$, the partition was lowered forcing the rat back into the waiting area. The trial was aborted and recorded as an omission. Progression to the next stage of the task occurred

Table I A Representative Order of Stimuli Used in an Attentional Set-shifting Task in which the Rat Must Shift from Odor to Digging Medium

\begin{tabular}{|c|c|c|c|c|}
\hline \multirow{2}{*}{ Discriminations } & \multicolumn{2}{|c|}{ Dimensions } & \multicolumn{2}{|c|}{$\begin{array}{l}\text { Exemplar } \\
\text { Combinations }\end{array}$} \\
\hline & Relevant & Irrelevant & $(+)$ & $(-)$ \\
\hline Simple (SD) & Odor & & OI & $\mathrm{O} 2$ \\
\hline \multirow[t]{2}{*}{ Compound (CD) } & Odor & Medium & OI/MI & $\mathrm{O} 2 / \mathrm{M} 2$ \\
\hline & & & OI/M2 & $\mathrm{O} 2 / \mathrm{MI}$ \\
\hline \multirow[t]{2}{*}{ Reversal (REVI) } & Odor & Medium & O2/MI & $\mathrm{Ol} / \mathrm{M} 2$ \\
\hline & & & $\mathbf{0 2} / \mathrm{M} 2$ & $\mathrm{Ol} / \mathrm{MI}$ \\
\hline \multirow{2}{*}{ Intradimensional shift (ID) } & Odor & Medium & 03/M3 & O4/M4 \\
\hline & & & O3/M4 & $\mathrm{O} 4 / \mathrm{M3}$ \\
\hline \multirow[t]{2}{*}{ Reversal (REV2) } & Odor & Medium & 04/M3 & $\mathrm{O} 3 / \mathrm{M} 4$ \\
\hline & & & 04/M4 & $\mathrm{O} / \mathrm{M3}$ \\
\hline \multirow[t]{2}{*}{ Extradimensional shift (ED) } & Medium & Odor & M5/O5 & M6/O6 \\
\hline & & & M5/O6 & M6/O5 \\
\hline \multirow[t]{2}{*}{ Reversal (REV3) } & Medium & Odor & M6/O5 & $\mathrm{M} 5 / \mathrm{O} 6$ \\
\hline & & & M6/O6 & M5/O5 \\
\hline \multicolumn{5}{|c|}{$\begin{array}{l}\text { Discrimination stages were presented in the order provided for every animal } \\
\text { starting with the SD and ending with the REV } 3 \text {. However, the assignment of } \\
\text { relevant or irrelevant dimension, rewarded }(+) \text { or unrewarded }(-) \text { stimuli, and } \\
\text { their position in the left-right testing arms in the testing environment was } \\
\text { pseudorandomized and counterbalanced across all subjects. With the exception } \\
\text { of the SD, each trial presented two pots that differed on two dimensions. The } \\
\text { rewarded exemplar appears in bold italics and is paired with both irrelevant } \\
\text { exemplars. See Table } 2 \text { for a list of the potential six exemplars representing each } \\
\text { of the three stimulus categories. }\end{array}$} \\
\hline
\end{tabular}

once criterion performance (six consecutive correct responses) was achieved.

The compound discrimination (CD) stage was performed using the same pots from the SD. The rewarded dimension remained the same but a second, irrelevant dimension was introduced. After criterion was reached for the $\mathrm{CD}$, the reinforcement rules were reversed (REV1) and the rat must learn that the previously correct stimulus within the rewarded dimension is now incorrect. This was true for all rule-reversal stages of the task (REV1, REV2, and REV3). After subjects achieved criterion performance on REV1, they were tested on the intra-dimensional shift (ID). During the ID test, the rats were presented with a novel set of stimuli but were required to attend to the same perceptual dimension that had been reinforced during the SD, $C D$, and REV1. The rats then received a reversal of ID rules (REV2) followed by testing of an ED. During the ED stage, novel stimuli were presented again; however, the rat had to inhibit responding to the previously relevant dimension as it was no longer rewarding and shift attention to the previously irrelevant dimension that was now reinforced. After criterion was reached for the ED, the final stage of the task included a reversal of ED rules (REV3).

All dimensions and pairs of exemplars (see Table 2 for exemplars used) were equally represented within groups and counterbalanced between groups to the extent possible. Thus, attentional set-shift testing was completed using six possible combinations of stimulus dimensions. These included shifts from odor to texture, odor to digging medium, texture to odor, texture to digging medium, digging medium to odor, and digging medium to texture.

\section{Drug Administration}

Subsets of nVHTTX and nVHSham rats were randomly assigned to receive the selective $\alpha 7 \mathrm{nAChR}$ partial agonist SSR180711 (Biton et al, 2007; Pichat et al, 2007) or saline control injections. On the day of testing, SSR180711 was dissolved in saline and either SSR180711 (3 mg/kg, i.p.) or $\mathrm{pH}$-matched saline were administered to adult rats (PD56-80). Following the injection, animals were placed in a holding cage and remained undisturbed for $40 \mathrm{~min}$. After

Table 2 Perceptual Dimensions and Specific Stimuli Used in the Attentional Set-shifting Task

\begin{tabular}{lllll}
\hline Dimension & Training set & Pair $\mathbf{~}$ & Pair $\mathbf{2}$ & Pair $\mathbf{3}$ \\
\hline Odor & Lavender & Cinnamon & Gardenia & Rose \\
& Raspberry & Patchouli & Jasmine & Lilac \\
\multirow{4}{*}{ Medium } & White paper & Light foam & Plastic beads & Plastic buttons \\
& Green paper & Dark foam & Metallic beads & Gold buttons \\
\multirow{2}{*}{ Texture } & Paper & Velour fabric & Felt fabric & Yarn \\
& Parafilm & Cotton fabric & Cotton fabric & Cotton fabric
\end{tabular}

The exemplars used in the training set were not repeated during the testing session. Each testing session used three sets of digging pots that were presented as pairs during different stages in the task. Specifically, one set was used for the single discrimination (SD), compound discrimination (CD), and the first reversal (REVI). Another set was used for the intra-dimensional shift (ID) and second reversal (REV2). A third set was used for the extra-dimensional shift (ED) and the third reversal (REV3). The assignment of sets of stimuli to stage clusters was randomized among rats. 

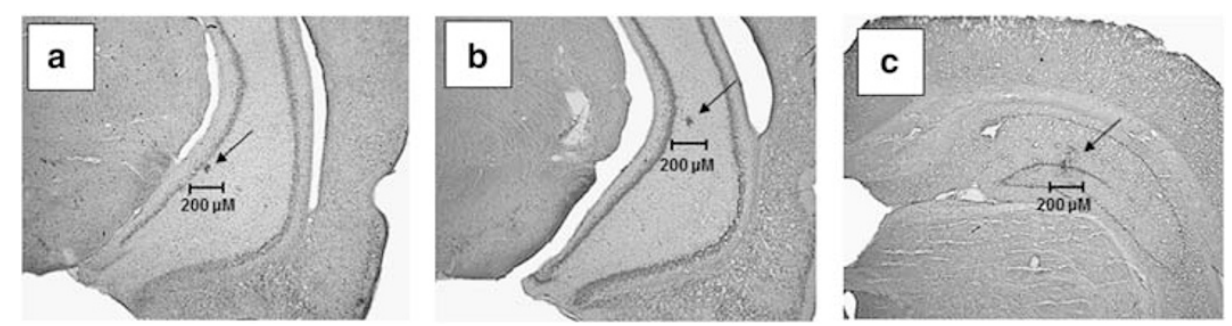

Figure I Nissl-stained coronal sections showing residual spots of dye indicating representative placements of tetrodotoxin infusions into the ventral hippocampus (CA3 region) on postnatal day 7 (PD7) (a), PD32 (b), and infusions into the dorsal hippocampus (dorsal dentate gyrus) on PD7 (c). All histological representations are from mature rats (>PD56).

this delay, animals were transferred into the testing apparatus to begin the seven discrimination stages. Drug doses and injection intervals were chosen based on our previous studies (Hansen et al, 2007; Pichat et al, 2007; Thomsen et al, 2009).

\section{Histological Procedures}

Following completion of Day 3 testing, animals were given an overdose of sodium pentobarbital and transcardially perfused with $0.9 \%$ heparinized saline followed by $10 \%$ formalin. The brain was extracted and placed in a $10 \%$ formalin bath and stored for $24 \mathrm{~h}$. The brain was then transported to a $30 \%$ sucrose solution bath and was refrigerated for at least 3 days. Once cryoprotected, brains were frozen, sectioned $(50 \mu \mathrm{m})$ with a cryostat, mounted on microscope slides, and stained. The center of the neonatal manipulation injection site was readily identified by the Evans Blue dye residue, which remained visible for months after local administration. Sections containing the injection site were stained using Nuclear Fast Red (Kernechtrot).

\section{Statistical Analyses}

Statistical analyses focused on the number of trials it took each rat to reach criterion at each discrimination stage. Initially, performance was analyzed in the VH PD7 groups using a mixed-design, two-way repeated measures ANOVA with discrimination STAGE as within-subjects measures and neonatal CONDITION as a between-subjects factor. Further comparisons were performed using one-way ANOVAs to examine sources of significant interactions. Finally planned $t$-tests were conducted comparing ID and ED shift performance when applicable. The analysis of the two control groups, the age- (VH PD32) and regional- (DH PD7) dependency of the cognitive deficits seen in the VHTTX PD7 group was conducted in a similar fashion. Significance was defined as alpha $=0.05$, and the HuynhFeldt correction was utilized to reduce type 1 errors associated with repeated measures ANOVAs (Vasey and Thayer, 1987). All statistical tests were performed using SPSS for windows (version 19.0; Chicago, IL).

\section{RESULTS}

\section{Verification of Saline and TTX Infusion Sites}

Figure 1 provides representative photomicrographs depicting placements of TTX infusion into the developing

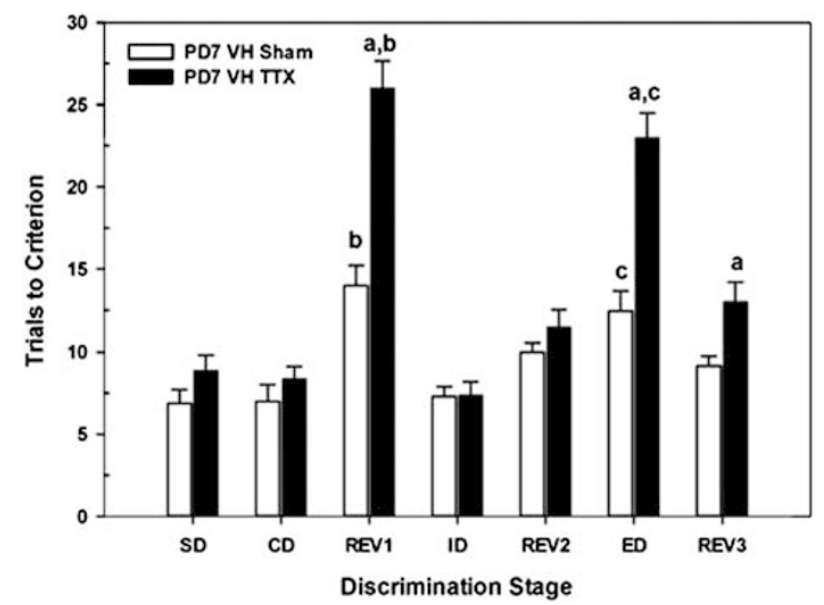

Figure 2 Mean number of trials to criterion ( \pm SEM) in the postnatal day 7 (PD7) ventral hippocampus $(\mathrm{VH})$ sham control $(n=7)$ and tetrodotoxin $(T T X)$ rats $(n=8)$ on each discrimination stage in the attentional set-shifting task. There were no differences among the groups in the acquisition of the task (simple discrimination (SD), compound discrimination (CD)), or in the intra-dimensional shift (ID) or REV2 stages. Rats receiving PD7 TTX inactivation of the $\mathrm{VH}$ required more trials to reach criterion performance on the REVI, extra-dimensional shift (ED), and REV3 stages than did the sham-control group. a=different from corresponding sham group at that respective test stage; $b=$ differences between CD and REVI stages within that treatment group; $c=$ differences between ED and ID stages within that treatment group; all P's $<0.05$.

hippocampus. Injection sites were revealed by discrete residues of Evans Blue Dye (deposited at the time of surgery) that remained visible at the time of testing (PD5680) for TTX and saline injections into the $\mathrm{VH}$ at PD7 (Figure 1a), PD32 (Figure 1b), and PD7 infusions into the DH (Figure 1c). Animals showing infusion sites outside the intended regions of the hippocampus were not included in the subsequent analyses.

\section{Effects of TTX-Induced Inactivation of the Neonatal VH on Attentional Set-Shifting}

Figure 2 summarizes task performance on the attentional set-shifting task for adults receiving intra- $\mathrm{VH}$ infusions of saline (VHSham) or TTX (VHTTX) on PD7. Relative to sham controls, TTX-treated rats exhibited selective deficits in task performance (STAGE $\times$ CONDITION, $F_{6,65}=10.21$, $P<0.001)$. To determine the stages of the task that were differentially affected by the inactivation, a series of oneway ANOVAs was conducted to assess the effects of 


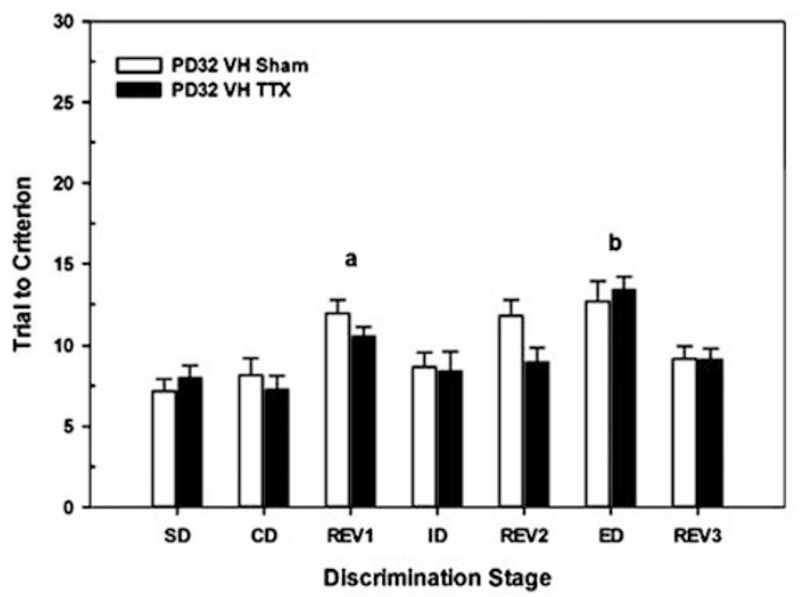

Figure 3 Mean number of trials to criterion ( \pm SEM) in postnatal day 32 (PD32) ventral hippocampus $(\mathrm{VH})$ sham control $(n=6)$ and tetrodotoxin (TTX) rats $(n=7)$ on each discrimination stage in the attentional setshifting task. Overall, there were differences among the various stages. However, there were no differences between the two groups in the acquisition of the task (simple discrimination (SD), compound discrimination (CD)), reinforcement reversals (REVI, REV2, REV3), or the test of intra-dimensional (ID) or extra-dimensional (ED) shifts in attention. $\mathrm{a}=$ difference between REVI and ED stages across sham and TTX groups; $\mathrm{b}=$ difference between ED and ID stages across sham and TTX group; both $P$ 's $<0.05$.

treatment condition on each stage of testing. TTX-induced impairments (ie, greater number of trials required to acquire task stage than in controls) were evident during REV1 $\left(F_{1,14}=32.36, P<0.001\right)$, ED $\left(F_{1,14}=29.20, P<0.001\right)$, and REV3 $\left(F_{1,14}=7.61, P=0.02\right)$. Performance within the remaining stages did not differ between the two groups (all $P$ 's $>0.05)$. As expected, acquisition of the initial reversal (REV1) required more trials than the previous $C D$ for shams $\left(t_{6}=-3.73, \quad P=0.01\right)$ and TTX-treated rats $\left(t_{7}=-8.90, P<0.001\right)$. Importantly, both groups of rats were able to learn an attentional set (the ID stage) that was restricted to the previously reinforced stimulus dimension of earlier stages. Finally, the ED required more trials than did the earlier intra-dimensional shift (ID) for both groups of rats (shams, $t_{6}=-3.32, \quad P=0.02$; TTX-treated, $\left.t_{7}=-10.18, P<0.001\right)$, with TTX-treated rats impaired relative to sham controls $(P<0.05)$.

Age-Dependent Effects of TTX-Induced Inactivation on Attentional Set-Shifting: Infusions into the VH on PD32

To determine whether there was a developmental sensitive period for the effects of $\mathrm{VH}$ inactivation, a group of rats received saline or TTX infusions into the VH on PD32. Figure 3 illustrates that there were overall differences in the trials to criteria among the various stages $\left(F_{6,66}=10.25\right.$, $P<0.001)$. For example, REV1 $>$ CD $\left(t_{12}=15.74, P<0.001\right)$ and $\mathrm{ED}>\mathrm{ID}\left(t_{12}=-4.49, P=0.001\right)$. However, unlike the effects at PD7, rats sustaining $\mathrm{VH}$ inactivation later in development exhibited control-like performance across the various stages of the set-shifting task (CONDITION, $\left.F_{1,11}=1.38, P=0.26\right)$.

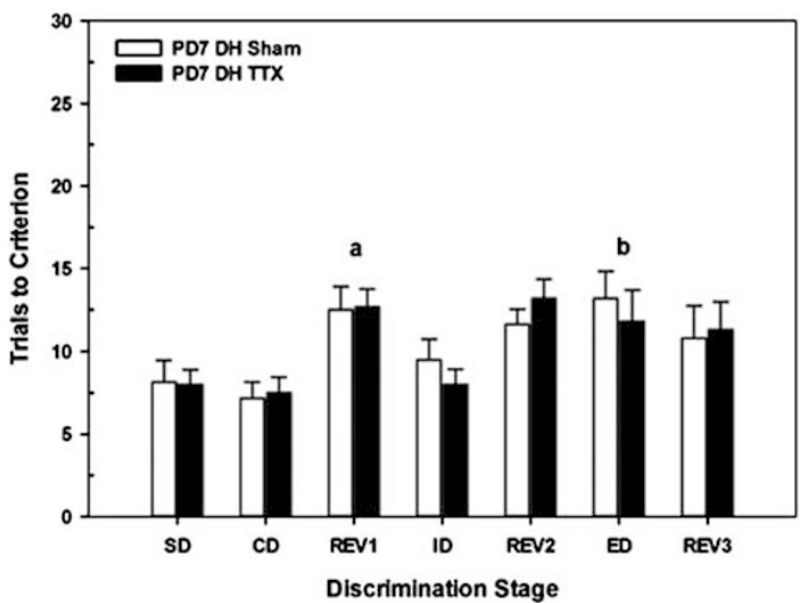

Figure 4 Mean numbers of trials to criterion ( \pm SEM) in postnatal day 7 (PD7) dorsal hippocampus $(\mathrm{DH})$ sham control $(n=6)$ and tetrodotoxin $(T \mathrm{TX})$ rats $(n=6)$ on each discrimination stage in the attentional setshifting task. Overall, there were differences among the various stages. However, there were no differences between the two groups in the acquisition of the task (simple discrimination (SD), compound discrimination (CD)), reinforcement reversals (REVI, REV2, REV3), or the test of intra-dimensional shift (ID) or extra-dimensional (ED) shifts in attention. $\mathrm{a}=$ difference between REVI and ED stages across sham and TTX groups; $\mathrm{b}=$ difference between ED and ID stages across sham and TTX group; both $P^{\prime} s<0.05$.

Region-Selective Effects of TTX-Induced Inactivation on Attentional Set-Shifting: Infusions into the DH on PD7

TTX was infused into the DH, in a separate group of rats, to assess the regional selectivity of the effects seen following $\mathrm{VH}$ inactivation at PD7. Figure 4 summarizes set-shifting performance in adults infused with saline (DHSham) or TTX (DHTTX) into the DH on PD7. Overall, performance across stages varied $\left(F_{6,51}=8.33, P<0.001\right)$ but the performance of the TTX-treated rats was no different than that of Shams at any of the seven stages (STAGE $\times$ CONDITION, $F_{6,51}=0.44, P=0.82$ ). Overall, rats required more trials to complete the initial reversal than the $C D$ $\left(t_{11}=-5.74, P<0.001\right)$. Likewise, acquisition of the ED required more trials than did the ID $\left(t_{11}=-4.49, P=0.001\right)$.

To further highlight the differences between the effects of inactivation of $\mathrm{VH}$ and $\mathrm{DH}$ on subsequent set-shifting ability, a direct comparison with the data presented in Figures 2 and 3 was conducted in an omnibus ANOVA. Consistent with the regional selectivity of the transient inactivation, the effects of TTX treatment were highly dependent upon the region infused and the stage of the setshifting task (STAGE $\times$ CONDITION $\times$ REGION, $\left.F_{6,138}=5.40, P<0.001\right)$.

\section{Reversal of Attentional Set-Shifting Deficits with an $\alpha 7$ nAChR Agonist}

Figure 5 illustrates the effects of acute administration of saline or the partial $\alpha 7 \mathrm{nAChR}$ agonist SSR180711 $(3 \mathrm{mg} / \mathrm{kg})$ on task performance in adults that received saline (VHSham) or TTX (VHTTX) on PD7. Overall, there were group differences in the number of trials required to complete certain stages of the task (CONDITION 


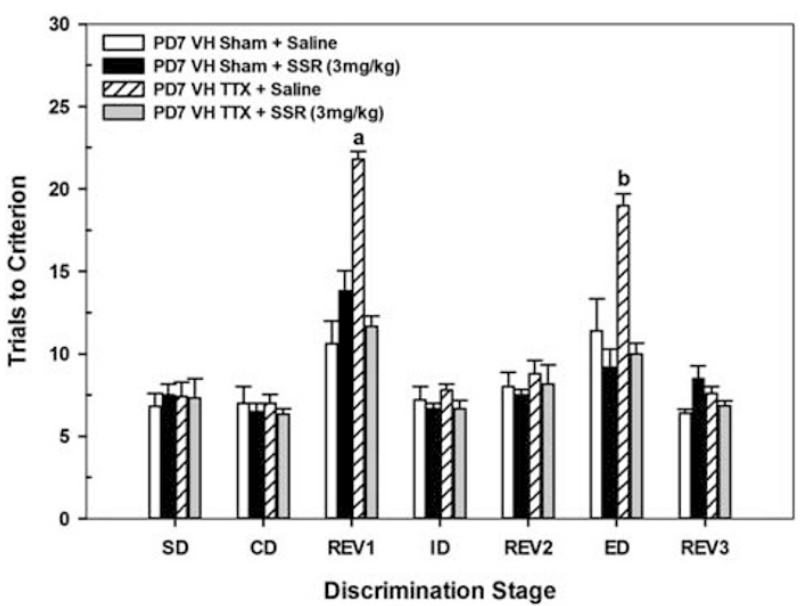

Figure 5 Mean number of trials to criterion $( \pm S E M)$ in postnatal day 7 (PD 7) $\mathrm{VH}$ sham control and tetrodotoxin (TTX) rats following acute systemic administration of saline (VH sham $n=6$; VHTTX $n=6)$ or the partial $\alpha 7$ nicotinic acetylcholine receptor agonist SSR I 807I I (3 mg/ $/ \mathrm{kg} ; \mathrm{VH}$ sham $n=6$; VHTTX $n=6$ ) at each discrimination stage of the attentional set-shifting task. Task performance was not affected by administration of saline or SSRI807II in sham control rats. Conversely, VHTTX rats receiving SSRI807I I showed improved task performance, comparable to that of controls, at the REVI and extra-dimensional shift (ED) stages. $a=$ different from each of the other treatment groups in REVI; $\mathrm{b}=$ different from each of the other treatment groups in ED; all P's $<0.05$.

$\times$ DRUG $\times$ STAGE, $\left.F_{6,108}=8.76, P<0.001\right)$. Two-way ANOVAs (DRUG $\times$ STAGE) revealed that administration of either saline or SSR180711 did not alter overall task performance in those rats treated with saline at $\operatorname{PD} 7\left(F_{4,32}=1.6\right.$, $P=0.24$ ) yet had considerable effects on performance in rats treated with TTX at PD7 $\left(F_{4,29}=16.7, P<0.001\right)$. A series of one-way ANOVAs, comparing the effects of saline and SSR 180711 at selective stages, was conducted in TTX-treated rats to identify the source(s) of this interaction. Rats treated with TTX on PD7 and given a control pre-task injection of saline exhibited elevated trials to criterion, relative to TTX rats treated with SSR 180711, in both the REV1 $\left(F_{1,10}=156.22, P<0.001\right)$ and ED stages $\left(F_{1,10}=\right.$ 90.37, $P<0.001)$. In fact, TTX rats treated with SSR 180711 performed no differently than sham rats treated with saline on any of the seven stages of the task (all P's $<0.05$ ).

\section{DISCUSSION}

The experiments described above revealed several important and novel observations. First, TTX-induced temporary inactivation of ventral hippocampal transmission at PD7 results in selective deficits in the initial reversal and ED stages of a set-shifting task. Second, these deficits were agedependent, as they were not evident following identical infusions later in development (periadolescence, PD32). Third, the deficits seen following VH infusions at PD7 were regionally selective within the hippocampal formation as TTX infusions into the DH at PD7 resulted in a performance profile that was similar to that of saline-treated controls. Finally, the task impairments induced by VHTTX at PD7 were completely reversed following an acute administration of a partial $\alpha 7 \mathrm{nAChR}$ agonist prior to testing in adults. In the sections that follow, we a) discuss potential mechanisms that could contribute to these age- and region-dependent TTX-induced deficits; b) speculate on the pro-cognitive effects of SSR180711 in reversing these performance deficits; and c) comment on the validity of TTX-induced $\mathrm{VH}$ inactivation as an animal model for studying deficits in cognitive flexibility seen in patients with SZ.

\section{Potential Mechanisms Contributing to Set-Shifting Deficits}

Rats receiving intra- $\mathrm{VH}$ infusions of TTX on PD7 exhibited normal rates of acquisition of the single and CDs suggesting that the inactivation did not produce significant differences in motivation, the ability to discriminate among the perceptual dimensions of the pots, or generalized deficits in learning the rules of the discrimination. Moreover, TTXtreated rats displayed the ability to form an attentional set as evidenced by their normal performance during the ID stage of the task. The primary deficits in these rats were the difficulty in learning the initial reversal (REV1) and in performing an ED.

The literature from humans, primates, and rodents (Dalley et al, 2004; Ghods-Sharifi et al, 2008; Rygula et al, 2010) indicates a critical role for the orbitofrontal cortex (OFC) in mediating the behavioral flexibility required for reversal learning, whereas the integrity of the PFC (Birrell and Brown, 2000; Robbins, 2007; Stefani and Moghaddam, 2005), with modulation from the NAC (Floresco et al, 2006), determines the ability to learn an ED shift. Efferents from the subicular region of the $\mathrm{VH}$ project directly onto interneuron and pyramidal target neurons in the PFC (Jay et al, 1989; Tierney et al, 2004) or indirectly to PFC via projections to NAC (French and Totterdell, 2003; Miller et al, 2010) and then through BF (Henny and Jones, 2008) or medial dorsal thalamus (Sarter \& Markowitsch, 1984). Intra$\mathrm{VH}$ infusions of TTX on PD7, but not on PD32, markedly disrupted the subsequent ability of rats to perform the initial reversal and ED stages of a set-shifting task. This age dependency suggests that neurodevelopmental events critical to the functional maturation of the PFC-, OFC-, NAC-, and BF-distributed system are ongoing during the first postnatal week, but are more mature and stable, at least for performance of the set-shifting task, later in development. Several mechanisms could account for these early developmental effects. First, the TTX infusions on PD7 may produce functional deficits in developing local GABAergic interneurons that regulate the maturation of the abovementioned VH efferents to PFC and NAC (Francois et al, 2009; Lipska et al, 2003). TTX infusions at later ages may fall outside of a critical window for target maturation as these inputs are establishing synaptic contacts in cortex from PD11 to PD20 (Sutor and Luhmann, 1995). Second, early exposure to TTX may restrict the maturation of these contacts and subsequent information flow by reducing spine density in frontal cortex. In cultured granule cells, the formation of mature dendritic spines was reduced following exposure to TTX (Drakew et al, 1999). These results are consistent with a reduction in cortical and hippocampal spine density in the brains of patients with SZ (Lewis and Gonzalez-Burgos, 2008). Third, we have previously reported (Brooks et al, 2011) that VH TTX infusions on PD7, but not PD32, disrupt the mesolimbic regulation of evoked 
prefrontal ACh levels. This dysregulation may contribute to the performance deficits in the task given the significant role that cortical cholinergic transmission has in attentional processing (Hasselmo and Sarter, 2011; Sarter et al, 2005) and, more specifically, the importance of the mesolimbic recruitment of prefrontal $\mathrm{ACh}$ release to maintain performance as the conditions of the task become more challenging (St Peters et al, 2011). Finally, in a series of studies using infusions of TTX into VH at PD7, Louilot and colleagues have reported abnormalities in accumbens dopaminergic transmission related to deficits in performance in a latent inhibition task (Meyer et al, 2009; Peterschmitt et al, 2008). Deficits in task-evoked accumbens DA release may contribute to impaired performance in the set-shifting task (Floresco et al, 2009; Goto and Grace, 2005).

In addition to the age dependency discussed above, the behavioral effects of TTX were markedly influenced by the region of the developing hippocampus in which the infusions were made. Unlike the case following TTX infusions into the $\mathrm{VH}$ at PD7, infusions into $\mathrm{DH}$ did not result in performance deficits. This regional difference between the cognitive effects of transient inactivation of the $\mathrm{VH} v s \mathrm{DH}$ directly parallels our recent report that infusions into the $\mathrm{VH}$, but not the $\mathrm{DH}$, produce deficits in the mesolimbic regulation of evoked prefrontal ACh levels (Brooks et al, 2011). The preservation of this modulation even after inactivation of the DH may contribute to the control-like performance in the task. Functional distinctions between the dorsal and ventral subregions of the hippocampus persist through adulthood. Using local infusions of NMDA antagonists in adult rats, Otto and colleagues have demonstrated dissociable roles of the two subregions in other forms of learning including, trace fear conditioning, contextual conditioning, and spatially guided alternation (Czerniawski et al, 2009, 2011).

\section{Cognition-Enhancing Effects of SSR180711}

The acute systemic administration of the partial $\alpha 7 \mathrm{nAChR}$ agonist SSR180711 (Biton et al, 2007) in adults, treated with TTX on PD7, reinstated the initial REV and ED deficits to control levels (Figure 5). Administration of SSR180711 has demonstrated pro-cognitive effects in other pharmacologically based animal models of SZ, including; novelty discrimination following neonatal PCP (Pichat et al, 2007); spatial working memory (Pichat et al, 2007) or latent inhibition (Barak et al, 2009) after acute MK801 administration (Barak et al, 2009); spatial working memory following semi-chronic administration of PCP (Thomsen et al, 2009); and attentional set-shifting after acute elevation of the $\alpha 7 \mathrm{nAChR}$ negative modulator kynurenic acid (Pershing et al, 2011).

The mechanisms underlying the cognition-enhancing effects of SSR180711 are not fully understood, although several possibilities have recently been suggested. Systemic administration of the same dose of SSR180711 $(3.0 \mathrm{mg} / \mathrm{kg})$ used in the present studies increases neuronal activity (elevations in c-Fos) in PFC, BF, and NAC (Hansen et al, 2007; Thomsen et al, 2010). The activation in PFC, but not $\mathrm{NAC}$, is dependent on the drug's stimulation of $\alpha 7 \mathrm{nAChRs}$ on cholinergic neurons in the $\mathrm{BF}$ projecting directly to the PFC (Thomsen et al, 2010). As expected, following the activation of the BF, SSR180711 enhanced ACh efflux in the PFC (Biton et al, 2007). Moreover, local infusion of SSR180711 into the PFC resulted in a significant phasic increase in glutamate release as measured by a glutamatesensitive biosensor (Bortz et al, 2011). This drug-induced increase in both prefrontal $\mathrm{ACh}$ and glutamate may contribute significantly to the normalization of performance in the REV and ED stages, respectively.

\section{TTX-Induced Inactivation of Developing VH as an Animal Model of SZ}

The transient inactivation of the VH during early development may enjoy a greater degree of face, construct, and predictive validity as an animal model of the cognitive deficits associated with SZ than current models employing permanent lesions. A consistent neuropathology in SZ is the subtle disruption of the anterior aspect of the hippocampal formation (Goldman and Mitchell, 2004; Harrison, 2004; Tamminga et al, 2010). While the specific, persistent chemoanatomical disruptions in the maturation of the hippocampus and its target regions following TTX on PD7 remain to be determined, they are likely to be more representative than the large, gross lesions produced by early ibotenic acid lesions of the $\mathrm{VH}$.

Many of the cognitive deficits seen in SZ appear to be mediated by dysregulated interactions between the hippocampus and its principal target regions, such as PFC (Henseler et al, 2010; Wolf et al, 2009), NAC (Chambers et al, 2001; Csernansky et al, 1993), and ventral tegmental area (VTA; Yang et al, 1999; Lisman et al, 2010). Thus, manipulations that alter the maturation of the $\mathrm{VH}$ (rodent counterpart of anterior hippocampal formation; Dolorfo and Amaral, 1998) through transient inactivation represent a rational approach for modeling at least two characteristic cognitive deficits seen in SZ. First, patients with SZ exhibit characteristic deficits in cognitive flexibility (Pantelis et al, 1999; Leeson et al, 2009) that can be approximated in animal models using REV/ID/ED tasks such as the setshifting task employed in the present experiment (Robbins, 2007; Stefani and Moghaddam, 2005; Tait et al, 2009). Second, SZ is also characterized by deficits in latent inhibition (Gray, 1998; Young et al, 2005; Schmidt-Hansen et al, 2009). Rats receiving TTX infusions into entorhinal cortex or ventral subiculum as neonates, but not as adults, exhibit deficits in latent inhibition (Meyer et al, 2009; Peterschmitt et al, 2007). Moreover, these deficits in latent inhibition are accompanied by a disruption of the normal coupling between performance in the task and striatal dopamine release (Meyer et al, 2009).

Finally, the VH transient inactivation animal model exhibits some degree of predictive validity toward the treatment of cognitive disorders in SZ. The beneficial effects of SSR180711 in our animal model are consistent with several recent reports, in patients with SZ, of modest cognition-enhancing properties of $\alpha 7 \mathrm{nAChR}$ agonists in tasks that do not involve an ID/ED shift (Buchanan et al, 2008; Martin and Freedman, 2007; Olincy et al, 2006; Schubert et al, 2006; Tregellas et al, 2011; but see Lindenmayer \& Khan (2011) for conflicting results). Collectively, these studies suggest that transient inactivation of the developing $\mathrm{VH}$ represents a useful animal model for 
the study of cognitive deficits in SZ and that drugs designed to enhance activity at $\alpha 7 \mathrm{nAChRs}$ may represent rational targets for the development of adjunctive medication for treating this disorder.

\section{ACKNOWLEDGEMENTS}

This research was supported by a research grant from the National Institute of Health (MH057436) to JPB and MS as well as the Danish Strategic Research Council (Cognito) and the NOVO Nordisk Foundation to JDM. The authors are grateful to Dr Dan Peters at NeuroSearch A/S for providing SSR 180711.

\section{DISCLOSURE}

Jens Mikkelsen has received honoraria for speaking at Pfizer Inc., and has received research support from Eli Lilly \& Co., H. Lundbeck A/S, and Neurosearch A/S. Martin Sarter has received honoraria for speaking at Lilly UK, and has received research support from Abbott Laboratories and Pfizer Pharmaceuticals. The other authors declare no conflict of interest.

\section{REFERENCES}

Alexander KS, Brooks JM, Sarter M, Bruno JP (2009). Disruption of mesolimbic regulation of prefrontal cholinergic transmission in an animal model of schizophrenia and normalization by chronic clozapine treatment. Neuropsychopharmacology 34: 2710-2720.

Barak S, Arad M, De Levie A, Black MD, Griebel G, Weiner I (2009). Pro-cognitive and antipsychotic efficacy of the alpha7 nicotinic partial agonist SSR180711 in pharmacological and neurodevelopmental latent inhibition models of schizophrenia. Neuropsychopharmacology 34: 1753-1763.

Birrell JM, Brown VJ (2000). Medial frontal cortex mediates perceptual attentional set shifting in the rat. J Neurosci 20: 4320-4324.

Biton B, Bergis OE, Galli F, Nedelec A, Lochead AW, Jegham S et al (2007). SSR180711, a novel selective alpha7 nicotinic receptor partial agonist: (I) binding and functional profile. Neuropsychopharmacology 32: 1-16.

Bortz DM, Brooks JM, Sarter M, Bruno JP (2011). Program No. 368.02. 2011 Neuroscience Meeting Planner, Society for Neuroscience: Washington, DC.

Brady AM (2009). Neonatal ventral hippocampal lesions disrupt set-shifting in adult rats. Behav Brain Res 205: 294-298.

Brady AM, Saul RD, Wiest MK (2010). Selective deficits in spatial working memory in the neonatal ventral hippocampal lesion rat model of schizophrenia. Neuropharmacology 59: 605-611.

Brooks JM, Sarter M, Bruno JP (2007). D2-like receptors in nucleus accumbens negatively modulate acetylcholine release in prefrontal cortex. Neuropharmacology 53: 455-463.

Brooks JM, Sarter M, Bruno JP (2011). Transient inactivation of the neonatal ventral hippocampus permantly disrupts the mesolimbic regulation of prefrontal cholinergic transmission: implications for schizophrenia. Neuropsychopharmacology 36: 2477-2487.

Buchanan RW, Conley RR, Dickinson D, Ball MP, Feldman S, Gold JM.et al (2008). Galantamine for the treatment of cognitive impairments in people with schizophrenia. Am J Psychiatry 165: 82-89.
Chambers RA, Krystal JH, Self DW (2001). A neurobiological basis for substance abuse comorbidity in schizophrenia. Biol Psychiaty 50: 71-83.

Chambers RA, Moore J, McEvoy JP, Levin ED (1996). Cognitive effects of neonatal hippocampal lesions in a rat model of schizophrenia. Neuropsychopharmacology 15: 587-594.

Csernansky JG, Wrona CT, Bardgett ME (1993). Subcortical dopamine and serotonin turnover during acute and subchronic administration of typical and atypical neuroleptics. Psychopharmacology 110: 145-151.

Czerniawski J, Ree F, Chia C, Otto T (2011). Dorsal vs ventral hippocampal contributions to trace and contextual conditioning: differential effects of regionally selective nmda receptor antagonism on acquisition and expression. Hippocampus; e-pub ahead of print 19 December 2011.

Czerniawski J, Yoon T, Otto T (2009). Dissociating space and trace in dorsal and ventral hippocampus. Hippocampus 19: 20-32.

Dalley JW, Cardinal RN, Robbins TW (2004). Prefrontal executive and cognitive functions in rodents: neural and neurochemical substrates. Neurosci Biobehav Rev 28: 771-784.

Dolorfo CL, Amaral DG (1998). Entorhinal cortex of the rat: organization of intrinsic connections. J Comp Neurol 398: 49-82.

Drakew A, Frotscher M, Heimrich B (1999). Blockade of neuronal activity alters spine maturation of dentate granule cells but not their dendritic arborization. Neuroscience 94: 767-774.

Flores G, Alquicer G, Silva-Gomez AB, Zaldivar G, Stewart J, Quirion $\mathrm{R}$ et al (2005). Alerations in dendritic morphology of prefrontal cortical and nucleus accumbens neurons in postpubertal rats after neonatal excitotoxic lesions of the ventral hippocampus. Neuroscience 133: 463-470.

Floresco SB, Ghods-Sharifi S, Vexelman C, Magyar O (2006). Dissociable roles for the nucleus accumbens core and shell in regulating set shifting. J Neurosci 26: 2449-2457.

Floresco SB, Zhang Y, Enomoto T (2009). Neural circuits subserving behavioral flexibility and their relevance to schizophrenia. Behav Brain Res 204: 396-409.

Francois J, Ferrandon A, Koning E, Angst M-J, Sander G, Nehling A (2009). Selective reorganization of GABAergic transmission in neonatal ventral hippocampal-lesioned rats. Int J Neuropsychopharmacol 12: 1097-1110.

French SJ, Totterdell S (2003). Individual nucleus accumbensprojection neurons receive both basolateral amysdala and ventral subicular afferents in rats. Neuroscience 119: 19-31.

Ghods-Sharifi S, Haluk DM, Floresco SB (2008). Differential effects of inactivation of the orbitofrontal cortex on strategy set-shifting and reversal learning. Neurobiol Learn Mem 89: 567-573.

Goldman MB, Mitchell CP (2004). What is the functional significance of hippocampal pathology in schizophrenia? Schizophr Bull 30: 367-392.

Goto Y, O’Donnell P (2002). Delayed mesolimbic system alteration in a developmental animal model of schizophrenia. J Neurosci 22: 9070-9077.

Goto Y, Grace AA (2005). Dopaminergic modulation of limbic and cortical drive of nucleus accumbens in goal-directed behavior. Nat Neurosci 8: 805-812.

Gray JA (1998). Integrating Schizophrenia. Schizophr Bull 24: 249-266.

Grecksch G, Bernstein HG, Becker A, Hollt V, Bogerts B (1999). Disruption of latent inhibition in rats with postnatal hippocampal lesions. Neuropsychopharmacology 20: 525-532.

Green MF, Kern RS, Heaton RK (2004). Longitudinal studies of cognition and functional outcome in schizophrenia: implications for MATRICS. Schizophr Res 72: 41-51.

Groenewegen H, Vermeulenvanderzee E, Dortschot A, Witter M (1987). Organization of the projections from the subiculum to the ventral striatum in the rat- A study using anterograde transport of phaseolus-vulgaris leukoagglutinin. Neuroscience 23: 103-120. 
Hansen HH, Timmermann DB, Peters D, Walters C, Damai MI, Mikkelsen JD (2007). Alpha-7 nicotinic acetylcholine receptor agonists selectively activate limbic regions of the rat forebrain: an effect similar to antipsychotics. J Neurosci Res 85: $1810-1818$.

Harrison PJ (2004). The hippocampus in schizophrenia: a review of the neuropathological evidence and its pathophysiological implications. Psychopharmacology 174: 151-162.

Hasselmo ME, Sarter M (2011). Modes and models of forebrain cholinergic neuromodulation of cognition. Neuropsychopharmacology 36: 52-73.

Henny P, Jones BE (2008). Projections from basal forebrain to prefrontal cortex comprise cholinergic, GABAergic and glutamatergic inputs to pyramidal cells or interneurons. Eur $J$ Neurosci 27: 654-670.

Henseler I, Falkai P, Gruber O (2010). Disturbed functional connectivity within brain networks subserving domain-specific subcomponents of working memory in schizophrenia: relation to performance and clinical symptoms. J Psychiatric Res 44: 364-372.

Jay TM, Glowinski J, Thierry AM (1989). Selectivity of the hippocampal projection to the prelimbic area of the prefrontal cortex in the rat. Brain Res 505: 337-340.

Keefe RS (2007). Cognitive deficits in patients with schizophrenia: effects and treatments. J Clin Psychiatry 68(Suppl 14): 8-13.

Kehagia AA, Murray GK, Robbins TW (2010). Learning and cognitive flexibility: frontostriatal function and monoaminergic modulation. Curr Opin Neurobiol 20: 199-204.

Kerns JG, Nuechterlein KH, Braver TS, Barch DM (2008). Executive functioning component mechanisms and schizophrenia. Biol Psychiatry 64: 26-33.

Laplante F, Srivastava LK, Quirion R (2004). Alterations in dopaminergic modulation of prefrontal cortical acetylcholine release in post-pubertal rats with neonatal ventral hippocampal lesions. J Neuroch 89: 314-323.

Leeson VC, Robbins TW, Matheson E, Hutton SB, Ron MA, Barnes TRE et al (2009). Discrimination learning, reversal, and setshifting in first-episode schizophrenia: stability over six years and specific associations with medication type and disorganization syndrome. Biol Psychiatry 66: 586-593.

Le Pen G, Moreau JL (2002). Disruption of prepulse inhibition of startle reflex in a neurodevelopmental model of schizophrenia: reversal by clozapine, olanzapine and risperidone but not by haloperidol. Neuropsychopharmacolog 27: 1-11.

Lewis DA, Gonzalez-Burgos G (2008). Neuroplasticity of neocortical circuits in schizophrenia. Neuropsychopharmacology 33: 141-165.

Lindenmayer JP, Khan A (2011). Galantamine augmentation of long-acting injectable risperidone for cognitive impairments in chronic schizophrenia. Schizophr Res 125: 267-277.

Lipska BK, Aultman JM, Verma A, Weinberger DR, Moghaddam B (2002a). Neonatal damage of the ventral hippocampus impairs working memory in the rat. Neuropsychopharmacology 27: 47-54.

Lipska BK, Halim ND, Segal PN, Weinberger DR (2002b). Effects of reversible inactivation of the neonatal ventral hippocampus on behavior in the adult rat. J Neurosci 22: 2835-2842.

Lipska BK, Lerman DN, Khaing ZZ, Weinberger DR (2003). The neonatal ventral hippocampal lesion model of schizophrenia: effects on dopamine and GABA mRNA markers in the rat midbrain. Eur J Neurosci 18: 3097-3104.

Lipska BK, Weinberger DR (2002). A neurodevelopmental model of schizophrenia: neonatal disconnection of the hippocampus. Neurotox Res 4: 469-475.

Lisman JE, Pi HJ, Zhang YC, Otmakhova NA (2010). A thalamohippocampal-ventral tegmental area loop may produce the positive feedback that underlies the psychotic break in schizophrenia. Bio Psychiatry 68: 17-24.
Marquis JP, Goulet S, Dore FY (2008). Neonatal ventral hippocampus lesions disrupt extra-dimensional shift and alter dendritic spine density in the medial prefrontal cortex of juvenile rats. Neurobiol Learn Mem 90: 339-346.

Martin LF, Freedman R (2007). Schizophrenia and the alpha7 nicotinic acetylcholine receptor. Int Rev Neurobiol 78: 225-246.

Meyer F, Peterschmitt Y, Louilot A (2009). Postnatal functional inactivation of the entorhinal cortex or ventral subiculum has different consequences for latent inhibition-related striatal dopaminergic responses in adult rats. Eur J Neurosci 29: 2035-2048.

Miller EK, Cohen JD (2001). An integrative theory of prefrontal cortex function. Annu Rev Neuroscience 24: 167-202.

Miller EJ, Saint Marie LR, Breier MR, Swerdlow NR (2010). Pathways from the ventral hippocampus and caudal amygdala to forebrain regions that regulate sensorimotor gating in the rat. Neuroscience 165: 601-611.

Nuechterlein KH, Luck SJ, Lustig C, Sarter M (2009). CNTRICS final task selection: control of attention. Schizophr Bull 35: 182-196.

O'Donnell P, Grace AA (1995). Synaptic interactions among excitatory afferent to nucleus accumbens neurons: Hippocampal gating of prefrontal cortical input. J Neurosci 15: 3622-3693.

O'Donnell P, Lewis BL, Weinberger DR, Lipska BK (2002). Neonatal hippocampal damage alters electrophysiological properties of prefrontal cortical neurons in adult rats. Cereb Cortex 12: 975-982.

Olincy A, Harris JG, Johnson LL, Pender V, Kongs S, Allensworth $D$ et al (2006). Proof-of-concept trial of an alpha7 nicotinic agonist in schizophrenia. Arch Gen Psychiatry 63: 630-638.

Pantelis C, Barber FZ, Barnes TR, Nelson HE, Owen AM, Robbins TW (1999). Comparison of set-shifting ability in patients with chronic schizophrenia and frontal lobe damage. Schizophr Res 37: 251-270.

Pershing ML, Bortz DM, Alexander KS, Brooks JM, Mikkelsen JD, Thomsen MS et al (2011). Program No. 163.17. 2011 Neuroscience Meeting Planner, Society for Neuroscience: Washington, DC.

Peterschmitt Y, Meyer F, Louilot A (2007). Neonatal functional blockade of the entorhinal cortex results in disruption of accumbal dopaminergic responses observed in latent inhibition paradigm in adult rats. Eur J Neurosci 25: 2504-2513.

Peterschmitt Y, Meyer F, Louilot A (2008). Differential influence of the ventral subiculum on dopaminergic responses observed in core and dorsomedial shell subregions of the nucleus accumbens in latent inhibition. Neuroscience 154: 898-910.

Pichat P, Bergis OE, Terranova JP, Urani A, Duarte C, Santucci V et al (2007). SSR180711: (II) efficacy in experimental models predictive of activity against cognitive symptoms of schizophrenia. Neuropsychopharmacology 32: 17-34.

Robbins TW (2007). Shifting and stopping: fronto-striatal substrates, neurochemical modulation and clinical implications. Philos Trans R Soc Lond B Biol Sci 362: 917-932.

Rygula R, Walker SC, Clarke HF, Robbins TW, Roberts AC (2010). Differential contributions of the primate ventrolateral prefrontal and orbitofrontal cortex to serial reversal learning. J Neurosci 27: 14552-14559.

Sarter M, Hasselmo ME, Bruno JP, Givens B (2005). Unraveling the attentional functions of cortical cholinergic inputs: interactions between signal-driven and cognitive modulation of signal detection. Brain Res Brain Res Rev 48: 98-111.

Sarter M, Markowitsch HJ (1984). Collateral innervation of the medial and lateral prefrontal cortex by amygdaloid, thalamic and brain-stem neurons. J Comp Neurol 224: 445-460.

Schmidt-Hansen M, Killcross A, Honey RC (2009). Latent inhibition, learned irrelevance, and schizotypy: assessing their relationship. Cogn Neuropsychiatry 14: 11-29. 
TTX in developing ventral hippocampus impairs cognition JM Brooks et al

Schubert MH, Young KA, Hicks PB (2006). Galantamine improves cognition in schizophrenia patients stabilized on risperidone. Biol Psychiatry 60: 530-533.

Stefani MR, Moghaddam B (2005). Systemic and prefrontal cortical NMDA receptor blockade differentially affect discrimination learning and set-shift ability in rats. Behav Neurosci 119: 420-428.

St Peters M, Demeter E, Lustig C, Bruno JP, Sarter M (2011). Enhanced control of attention by stimulating mesolimbiccorticopetal cholinergic circuitry. J Neurosci 31: 9760-9771.

Sutor B, Luhmann HJ (1995). Development of excitatory and inhibitory postsynaptic potentials in the rat neocortex. Perspect Dev Neurobiol 2: 409-419.

Tait DS, Marston HM, Shahid M, Brown VJ (2009). Asenapine restores cognitive flexibility in rats with medial prefrontal cortex lesions. Psychopharmacology 202: 295-306.

Tamminga CA, Stan AD, Wagner AD (2010). The hippocampal formation in schizophrenia. Am J Psychiatry 167: 1178-1193.

Thomsen MS, Christensen DZ, Hansen HH, Redrobe JP, Mikkelsen JD (2009). Alpha(7) nicotinic acetylcholine receptor activation prevents behavioral and molecular changes induced by repeated phencyclidine treatment. Neuropharmacology 56: 1001-1009.

Thomsen MS, Hay-Schmidt A, Hansen HH, Mikkelsen JD (2010). Distinct neural pathways mediate alpha7 nicotinic acetylcholine receptor-dependent activation of the forebrain. Cereb Cortex 20: 2092-2102.

Tierney PL, Degentetais E, Thierry AM, Glowinski J, Gioanni Y (2004). Influence of the hippocampus on interneurons of the rat prefrontal cortex. Eur J Neurosci 20: 514-524.
Tregellas JR, Tanabe J, Rojas DC, Shatti S, Olincy A, Johnson L et al (2011). Effects of an alpha 7-nicotinic agonist on default network activity in schizophrenia. Biol Psychiatry 69: 7-11.

Tseng KY, Chambers RA, Lipska BK (2009). The neonatal ventral hippocampal lesion as a heuristic neurodevelopmental model of schizophrenia. Behav Brain Res 204: 295-305.

Vasey MW, Thayer JF (1987). The continuing problem of false positives in repeated measures ANOVA in psychophysiology- a multivariate solution. Psychophysiology 24: 479-486.

Wolf RC, Vasic N, Sambataro F, Hose A, Frasch K, Schmid M et al (2009). Temporally anticorrelated brain networks during working memory performance reveal aberrant prefrontal and hippocampal connectivity in patients with schizophrenia. Prog Neuro-Psychopharmacol Biol Psychiatry 33: 1464-1473.

Yang CR, Seamans JK, Gorelova N (1999). Developing a neuronal model for the pathophysiology of schizophrenia based on the nature of electrophysiological actions of dopamine in the prefrontal cortex. Neuropsychopharmacology 21: 161-194.

Young AM, Kumari V, Mehrotra R, Hemsley DR, Andrew C, Sharma T et al (2005). Dirsuption of learned irrelevance in acute schizophrenia in a novel continuous within-subject paradigm suitable for fMRI. Behav Brain Res 156: 277-288.

Zaborszky L, Cullinan WE (1992). Projections from the nucleus accumbens to cholinergic neurons of the ventral pallidum: a correlated light and electron microscopic double-immunolabeling study in rat. Brain Res 570: 92-101.

Zmarowski AL, Sarter M, Bruno JP (2005). NMDA and dopamine interactions in the nucleus accumbens modulate cortical acetylcholine release. Eur J Neurosci 22: 1731-1740. 